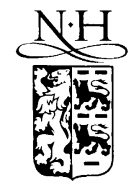

ELSEVIER

\title{
Buffer-layer-assisted nanostructure growth via two-dimensional cluster-cluster aggregation
}

\author{
Christina Haley, J.H. Weaver * \\ Department of Materials Science and Engineering, Frederick Seitz Materials Research Laboratory, University of Illinois at \\ Urbana-Champaign, 1304 West Green Street, Urbana, IL 61801, USA
}

Received 14 February 2002; accepted for publication 25 July 2002

\begin{abstract}
Physical vapor deposition of $\mathrm{Au}$ onto Xe multilayers on amorphous carbon at $20 \mathrm{~K}$ produces three-dimensional nanoclusters. Warming to room temperature desorbs the Xe and imparts limited mobility to the clusters. Coalescence occurs when two clusters come in contact, and the extent of coalescence depends on the buffer layer thickness. Using transmission electron microscopy images, we determined the spatial distribution of clusters as a function of Xe thickness and applied the scaling concepts of cluster-cluster aggregation to better understand processes associated with growth. Analysis shows a coverage dependent fractal dimension that extends from $D \sim 1.42$ to 1.72 for initial fractional surface coverages of $\rho_{0} \sim 0.04-0.21$, consistent with Monte Carlo simulations of two-dimensional diffusion-limited cluster aggregation (DLCA). Both the total number of clusters per unit area and the weighted average cluster size show a power law dependence on the Xe layer thickness, where the buffer layer thickness plays the role of time in DLCA modeling. These relationships will facilitate the design of nanostructure arrays generated by desorption-assisted coalescence.
\end{abstract}

(c) 2002 Elsevier Science B.V. All rights reserved.

Keywords: Electron microscopy; Evaporation and sublimation; Growth; Gold; Clusters

\section{Introduction}

In the last two decades, thorough studies of fractal cluster formation due to random particle and cluster aggregation have been carried out by means of Monte Carlo simulations [1-8]. Aggregation models have been created that describe quite well experimental systems such as smoke particle coagulation [9] and colloid aggregation

\footnotetext{
${ }^{*}$ Corresponding author. Tel.: +217-333-1440; fax: +217-3332736.

E-mail address: jhweaver@uiuc.edu (J.H. Weaver).
}

$[10,11]$. Recently, we introduced a new growth procedure, buffer-layer-assisted growth (BLAG), in which nanostructures of a wide variety of materials can be formed on a Xe multilayer condensed on a substrate [12]. Desorption of the Xe causes the nanostructures to move and to come into contact. Changing the thickness of the buffer layer, $t_{\mathrm{Xe}}$, makes it possible to control the size and density of the nanostructures that ultimately reach the surface. To better understand desorption-assisted coalescence, we apply scaling concepts for two-dimensional (2-D) cluster-cluster $(\mathrm{Cl}-\mathrm{Cl})$ aggregation to nanostructures resulting from BLAG. 
The $\mathrm{Cl}-\mathrm{Cl}$ aggregation model considers a 2-D lattice with a finite number of sites. A fraction of the sites are initially picked at random and occupied, giving an initial fractional surface coverage, $\rho_{0}$. Particles at nearest-neighbor sites are considered to belong to one cluster. Clusters and single particles are selected at random, moved by one or more lattice spacing(s) in a random direction, and merged if one contacts another (occupies a nearest neighbor site). Only one large aggregate remains in the limit of long time $[3,4]$. The probability that clusters merge differentiates the three most common $\mathrm{Cl}-\mathrm{Cl}$ aggregation models. A sticking probability of unity is used in diffusion-limited cluster aggregation (DLCA) and ballistic cluster aggregation (BCA) in which clusters diffuse via random walks and follow linear trajectories, respectively. The sticking probability is much less than unity for reaction-limited cluster aggregation (RLCA); as a result, clusters investigate numerous sticking configurations before one is chosen at random. RLCA permits greater cluster penetration into the growing structure, which results in a denser aggregate and a slower aggregation rate as compared to DLCA and BCA. Consequently, the geometric and dynamic properties of structures are specific to the method of aggregation. Structures formed via RLCA, for example, grow exponentially in time with a 2-D fractal dimension of approximately 1.55 while structures formed via DLCA grow with a power law dependence on time and exhibit a fractal dimension of only 1.44 in the low coverage limit [13-16].

In this paper, we analyze $\mathrm{Au}$ structures formed by BLAG using geometric and dynamic scaling techniques for $\mathrm{Cl}-\mathrm{Cl}$ aggregation. Using transmission electron microscopy (TEM) images, we determine the fractal dimension of structures with a correlation of the cluster area to the average radius. Dynamic behavior is considered in terms of the dependence of the average cluster size and density on the Xe buffer layer thickness. The results show that (i) BLAG nanostructures obey geometric scaling with a fractal dimension from $D \sim 1.42$ to 1.72 for $\rho_{0} \sim 0.04$ to 0.21 , and (ii) the weighted average cluster size grows as $S \sim t_{\mathrm{Xe}}^{z}$ while the density of structures decays as $N \sim t_{\mathrm{Xe}}^{-z}$ with $z \sim 2$. These results are consistent with 2-D
DLCA simulations in which a fractal dimension of $1.44-1.75$ is reported for $\rho_{0}$ from the dilute limit to $\sim 0.25$ [3,17], and dynamic behavior is described by a power law dependence on time. We conclude that $\mathrm{Xe}$ desorption-assisted coalescence can be described by 2-D DLCA, and the scaling expressions give predictive capabilities for the growth of nanostructures with BLAG.

\section{Experimental}

The nanostructures were prepared in an ultrahigh vacuum chamber equipped with a closed cycle $\mathrm{He}$ refrigerator and a cold finger. Amorphous carbon films (20-30 nm in thickness), supported on copper grids, were mounted directly onto the cold finger. After a brief bakeout, the typical chamber operating pressure was $\sim 2 \times 10^{-10}$ Torr, and the samples were cooled to $20 \pm 5 \mathrm{~K}$. Buffer layers of solid Xe were formed by exposure to 20 $1200 \mathrm{~L}$ of Xe $(99.995 \%)$ where $1 L=10^{-6}$ Torrs. The resulting $\mathrm{Xe}$ films ranged in thickness, $t_{\mathrm{Xe}}$, from approximately 4-215 ML. ${ }^{1}$ After Xe overlayer growth, the samples were exposed to a flux of $\mathrm{Au}$ atoms from a thermal source (deposition rate $\sim 0.6-1 \AA \mathrm{min}^{-1}$ ) resulting in a distribution of clusters on the Xe surface.

$\mathrm{Xe}$ desorption is negligible during $\mathrm{Au}$ atom impingement and cluster formation (even though the arrival energy of $\mathrm{Au}$ atoms from a $1350 \mathrm{~K}$ source is $\sim 0.18 \mathrm{eV}^{-1}$ atom $^{-1}$, the cohesive energy of bulk $\mathrm{Au}$ is $\sim 3.81 \mathrm{eV}$ atom $^{-1}$, and the cohesive energy of $\mathrm{Xe}$ is $\sim 0.17 \mathrm{eV}$ atom $^{-1}$ ) since the buffer layer is effective in transferring the thermal energy released. Pressure increases corresponding to desorption of more than 0.01 ML Xe were not observed during Au evaporation. Though stable, the buffer layer should not be envisioned as static during atom impingement and cluster formation since clusters that form are three-dimensional (3-D),

\footnotetext{
${ }^{1}$ Correcting the ion gauge reading for its sensitivity to $\mathrm{Xe}$ and assuming a sticking coefficient of unity at $20 \mathrm{~K}$ gives a Xe growth rate of $1 \mathrm{ML}$ per $\sim 5.5 \mathrm{~L}$ exposure, where $1 \mathrm{ML}=10^{15}$ atoms $\mathrm{cm}^{-2}$ and $1 \mathrm{~L}=10^{-6}$ Torrs.
} 
displace surface atoms, penetrate into the Xe surface, and are decorated with Xe. ${ }^{2,3}$

Following their formation, the clusters were delivered to the carbon substrate by warming the sample and desorbing the Xe. It is during this desorption period that clusters are mobile and coalescence can occur. The sample warmed at 7 $\mathrm{K} \mathrm{min}^{-1}$ from 20 to $50 \mathrm{~K}$ and at approximately 1 $\mathrm{K} \mathrm{min}^{-1}$ from $50 \mathrm{~K}$ to room temperature via black body radiation and back-side conduction when the closed cycle refrigerator was turned off. Xenon thermal desorption data show that significant desorption does not occur until the multilayer desorption temperature of $63 \pm 5 \mathrm{~K}$ is reached. With the current warm-up protocol, this occurs within $15 \mathrm{~min}$. The time interval for desorption depends on $t_{\mathrm{Xe}}$; it occurs over a period of approximately 5 min for a $60 \mathrm{ML}$ buffer layer. A faster warm-up procedure in which cold samples were removed from the cold head with a room temperature transfer arm generated similar cluster distributions [20].

The samples were ultimately removed from the growth chamber and imaged at room temperature with a Philips CM12 TEM operating at $120 \mathrm{kV}$. Imaging was typically undertaken within $48 \mathrm{~h}$, but a time delay is not important since the same cluster density and size distribution were not observed when imaging was undertaken $3 \mathrm{~h}$ or 1 year

\footnotetext{
${ }^{2}$ Waddill et al. [18] showed that the Xe $4 d$ photoemission features were broadened upon cluster formation. This reflects the fact that Xe would wet the clusters and adopt the vacuum level energy reference of the clusters. Broadening reflects the many inequivalent local work functions on the cluster/Xe surface.

${ }^{3}$ Ohno et al. [19] also reported the sinking of large Ag clusters with time due to attractive dispersion forces with the substrate. For a $10 \mathrm{ML}$ buffer at $50 \mathrm{~K}$, a time interval of $4 \mathrm{~h}$ was sufficient for $2 \mathrm{~nm}$ clusters to sink to the Xe-GaAs interface. This van der Waals attraction is most significant for thin $\mathrm{Xe}$ films ( 4-14 ML in thickness). For $t_{\mathrm{Xe}}=60 \mathrm{ML}$, we found that there were no changes in the final density or cluster size distribution, following $\mathrm{Xe}$ desorption, for clusters that were allowed to rest on the Xe surface at $20 \mathrm{~K}$ for intervals of $1 \mathrm{~min}$ to $10 \mathrm{~h}$. Were there significant sinking, then the effective thickness of the buffer layer would be smaller than $60 \mathrm{ML}$ and desorption-assisted coalescence would have been less effective.
}

after sample preparation. ${ }^{4}$ Hence, additional diffusion and aggregation of clusters on the amorphous carbon substrate is negligible. On the time scale of the TEM observations, we found no electronbeam-induced cluster rearrangement. This indicates that the effective conductivity of the system, including the contact between the nanostructures and the substrate, is sufficient to dissipate any energy absorbed from the electron beam.

\section{Results and discussion}

Fig. 1 shows the results of cluster rearrangement and coalescence following desorption of $t_{\mathrm{Xe}}=4-215 \mathrm{ML}$. In each case, the amount of deposited Au was $5 \AA$. Fig. 1(a) represents growth on the thinnest buffer layer studied, 4 ML. Previous STM studies [20] have shown that $4 \mathrm{ML} \mathrm{Xe}$ is sufficient to buffer the substrate. It is also thin enough that desorption does not significantly change the cluster size and distribution (there is not enough perturbation to cause aggregation). Accordingly, we take the size and density of clusters in Fig. 1(a) to represent the initial cluster distribution on the buffer for $5 \AA \mathrm{Au}$ depositions, regardless of $t_{\mathrm{Xe}}$. The density of clusters in Fig. (a) is $4.6 \times 10^{11} \mathrm{~cm}^{-2}$, and the average cluster radius is approximately $37 \AA$. The fraction of the Xe surface covered by clusters for $5 \AA \mathrm{Au}$ depositions is $\rho_{0} \sim 0.21$; the average nanostructure contains approximately 6300 atoms and has a height of $\sim 25 \AA$.

For comparison, Huang et al. [20] reported a cluster density of $1 \times 10^{12} \mathrm{~cm}^{-2}$ with an average cluster height of $6 \AA$ following depositions of $0.2 \AA$ $\mathrm{Ag}$ on $4 \mathrm{ML} \mathrm{Xe}$ on $\mathrm{Si}\left(\begin{array}{lll}1 & 1 & 1\end{array}\right)-7 \times 7$. As expected from nucleation theory [22], the cluster density varied as $N^{3}=3 R \vartheta / D$ where $N$ is the number density of stable islands, $R$ is the deposition rate, $\vartheta$ is the total coverage, and $D$ is the surface diffusion

\footnotetext{
${ }^{4}$ Chey et al. [21] studied the decay of Ag nanostructures delivered to $\operatorname{Ag}(111)$. In that case, time was an important parameter, and the decay of multilayer Ag islands could be followed over the course of several hours. Decay was facilitated by the low barrier for interlayer transport and the high diffusivity of $\mathrm{Ag}$ on $\mathrm{Ag}\left(\begin{array}{lll}1 & 1 & 1\end{array}\right)$ at room temperature.
} 

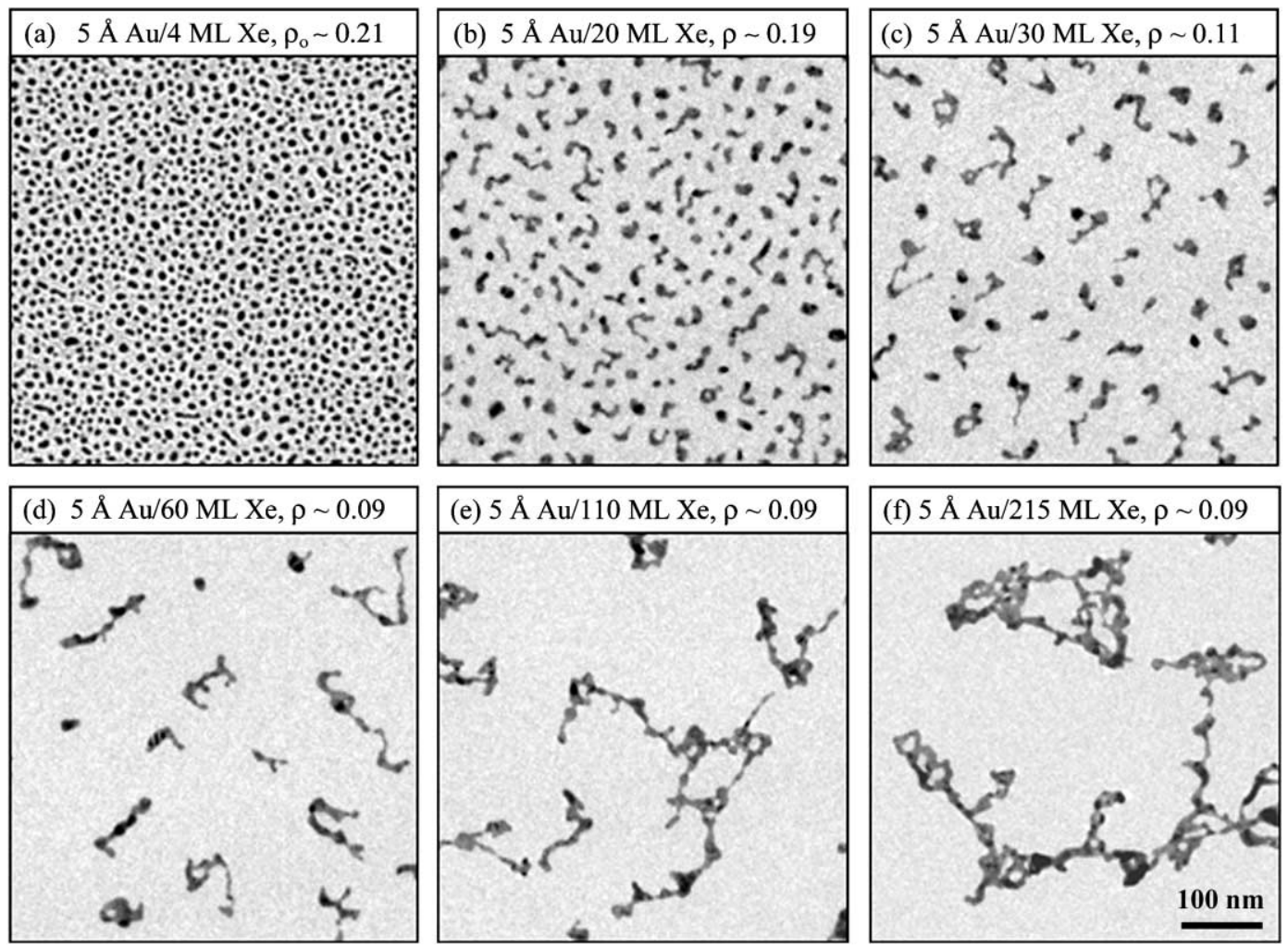

Fig. 1. Nanostructures produced when $5 \AA$ Au was deposited on buffer layers thicknesses, $t_{\mathrm{Xe}}$, of (a) $4 \mathrm{ML}$, (b) $20 \mathrm{ML}$, (c) $30 \mathrm{ML}$, (d) $60 \mathrm{ML}$, (e) $110 \mathrm{ML}$, and (f) $215 \mathrm{ML}$ on amorphous carbon at $20 \mathrm{~K}$. Imaging was done at room temperature. Desorption-assisted coalescence increases with $t_{\mathrm{Xe}}$ resulting in distributions of fractal structures. All images are $500 \times 500 \mathrm{~nm}^{2}$.

coefficient of adatoms. The weak dependence of the density on the amount deposited for $0.2-0.5 \AA \mathrm{Ag}$ reflects the slow deposition rate and an adatom mobility that was sufficient for most adatoms to reach existing nuclei. In our case, continued deposition to $5 \AA$ allows these islands to grow further, and their density decreases as coalescence occurs for those that are close together. Coalescence is confirmed by Fig. 1(a) which shows elongated islands with non-uniform thickness. One can speculate that the islands growing on a buffer of only $4 \mathrm{ML}$ thickness ultimately displace the $\mathrm{Xe}$ and make contact with the substrate, even before desorption.

Huang et al. [20] were the first to show that desorption-assisted coalescence increases with buffer layer thickness. Fig. 1(b) reveals that cluster coalescence for $5 \AA \mathrm{Au}$ and $20 \mathrm{ML}$ Xe reduces the density almost five-fold to approximately $1 \times 10^{11}$ $\mathrm{cm}^{-2}$. The fact that the surface covered by $\mathrm{Au}$ remains nearly constant at $\rho \sim 0.19$ indicates that coalescence results from motion that is largely parallel to the Xe surface. The result is that the structures in Fig. 1(a)-(b) have nearly constant heights of $\sim 25 \AA$. The irregular shapes reflect competition between thermodynamics, which favors equilibrium structures with minimal surface areas, and kinetics that imposes constraints due to low temperature and short times prior to contact with the carbon substrate.

Fig. 1(c)-(f) show the extent of cluster coalescence following desorption of 30,60, 110, and 215 ML of Xe. As can be seen, the morphologies are more irregular with lower surface coverage for $t_{\mathrm{Xe}}$ above $30 \mathrm{ML}$ in thickness. Desorption of these thicker buffer layers generates more cluster movement and larger structures. As a result, the density of structures decreases from $2.4 \times 10^{10}$ for $t_{\mathrm{Xe}}=30$ ML to $3.2 \times 10^{8} \mathrm{~cm}^{-2}$ for $t_{\mathrm{Xe}}=215 \mathrm{ML}$. 
From the reduced surface coverage, we infer a two-fold increase in height in Fig. 1(c)-(f) relative to Fig. 1(a)-(b), from $\sim 25$ to $50 \AA$. This is consistent with a two-fold increase in height in the same buffer layer thickness range for $8 \mathrm{~A} \mathrm{Au}$ deposited onto graphite. Using scanning tunneling microscopy, we measured nanostructure average height increases from approximately $50 \AA$ for 20 ML Xe to $100 \AA$ for $60 \mathrm{ML} \mathrm{Xe}$. The height increase suggests a transition that allows structure rearrangement during coalescence, i.e., such events occur far enough away from the substrate that structural arrangement is possible before impact. Additional rearrangement on a short length scale is evident from the thickness variations for the extended structures in Fig. 1(d)-(f). While these thickening events are outside the realm of conventional 2-D cluster-cluster aggregation models, a comparison with the models is useful to demonstrate that desorption-assisted coalescence is in accordance with random cluster aggregation.

\subsection{Fractal geometry}

The fractal dimension of structures formed by BLAG can be calculated with the relationship $s \propto R^{D}$ where $s$ is the area covered by each structure, $R$ is the average distance from the center of mass of a structure to its perimeter, and $D$ is the fractal dimension. Accordingly, a linear fit to a logarithmic plot of $s$ versus $R$ should yield the fractal dimension of an assembly of nanostructures. As a check of our procedure in determining $s$ and $R$, we analyzed 40 clusters from a digitized image of a 2-D DLCA Monte Carlo simulation from Ref. [17]. Our calculated fractal dimension was $1.76 \pm 0.02$, in agreement with the reported value of $1.75 \pm 0.07$ [17].

Analysis of the fractal dimension of BLAG nanostructures is presented in Fig. 2 for 4635 nanostructures generated by desorption-assisted coalescence for $5 \AA \mathrm{Au}$ depositions, as in Fig. 1(b)-(f). Linear behavior holds over two orders of magnitude in measured cluster size. A linear fit to the data, weighted in favor of the larger clusters, yields a fractal dimension of $D=1.72 \pm 0.03$. We have also analyzed the fractal dimension of nanostructures resulting from 1,3 , and $8 \AA \mathrm{Au}$ depo-

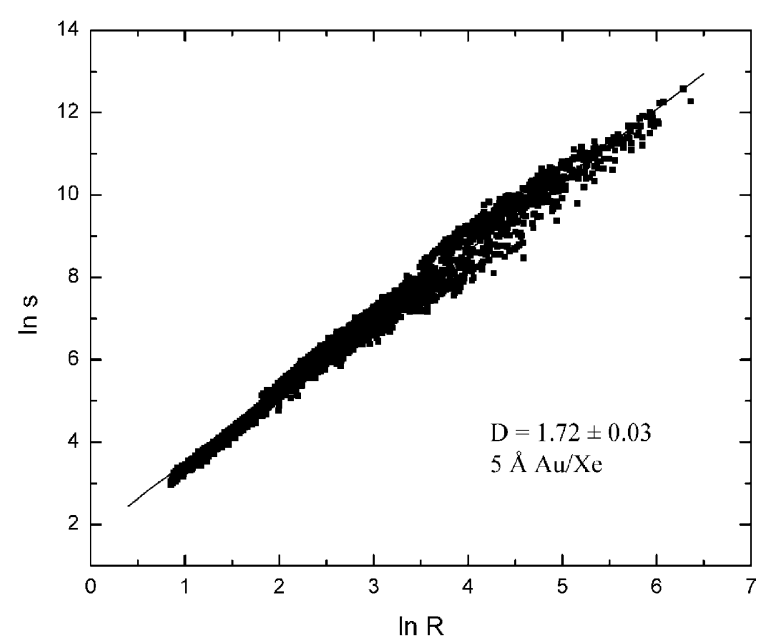

Fig. 2. Power law growth of the average cluster area, $s$, as a function of the average cluster radius, $R$, for structures in Fig. 1(b)-(f). The data represent a collection of 4635 nanostructures from 10 samples. The linear fit, weighted in favor of the larger structures, gives a fractal dimension of $1.72 \pm 0.03$.

sitions on $\mathrm{Xe}$ of varying thickness. From thin buffer layer experiments, like Fig. 1(a), the fraction of the Xe buffer covered by $\mathrm{Au}$ prior to cluster movement and coalescence was $\rho_{0} \sim 0.04,0.12$, and 0.23 for depositions of 1,3 , and $8 \AA$, respectively. In all cases, structures with irregular geometries like those in Fig. 1 were observed after Xe desorption. Linear fits to logarithmic plots of $s$ versus $R$ gave $D$ values of $1.42,1.59$, and 1.65 for depositions of 1,3 , and $8 \AA$, respectively.

A comparison of the fractal dimension of BLAG nanostructures to the three $\mathrm{Cl}-\mathrm{Cl}$ aggregation models is only possible in the limit of low coverage due to the limited literature for BCA and RLCA simulations. Table 1 summarizes fractal dimensions for $\rho_{0} \ll 1$ from experiment and from Monte Carlo simulations. The RLCA model gives the largest fractal dimension because the small sticking probability allows the greatest degree of cluster penetration into the growing structures. The DLCA model results in the least amount of penetration and the smallest fractal dimension. In the limit of small $\rho_{0}$, the fractal dimension for our BLAG nanostructures, $D=1.42 \pm 0.05$, is closest to that obtained with 2-D DLCA, $D=1.44 \pm 0.05$ $[3,13]$. 
Table 1

Fractal dimensions for $\rho_{0} \ll 1$ from experiment and from 2-D Monte Carlo simulations for diffusion-limited, ballistic, and reaction-limited cluster-cluster aggregation

\begin{tabular}{ll}
\hline & Fractal dimension \\
\hline Experiment $\left(\rho_{0} \sim 0.04\right)$ & $1.42 \pm 0.05$ \\
DLCA & $1.44 \pm 0.05[3,13]$ \\
BCA & $1.51 \pm 0.05[13]$ \\
RLCA & $1.55 \pm 0.05[13]$ \\
\hline
\end{tabular}

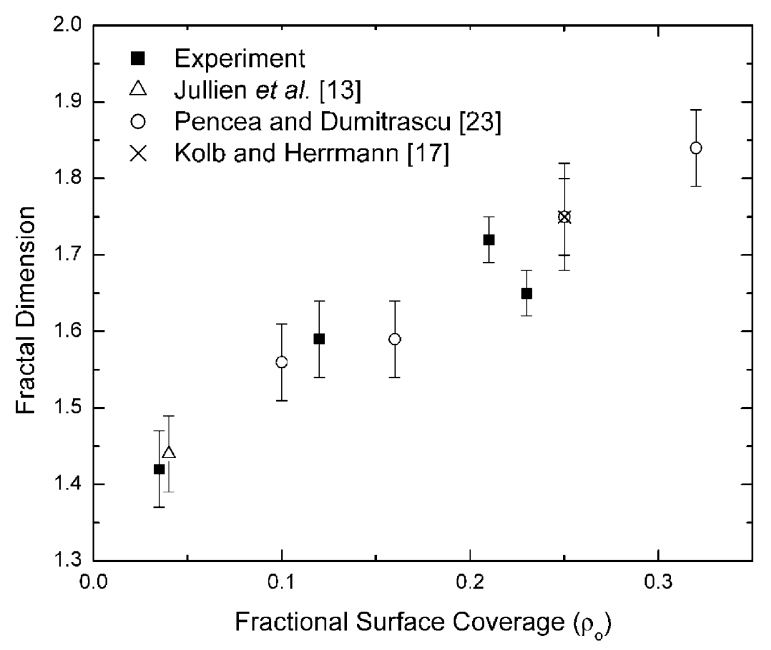

Fig. 3. Dependence of the fractal dimension on initial surface coverage, $\rho_{0}$. Filled squares represent our experimental results. Other symbols represent Monte Carlo simulations for 2-D DLCA. The increase in fractal dimension with coverage reflects the rapid growth of branches that capture smaller clusters.

Contrary to BCA and RLCA models, there are many DLCA simulations, including studies beyond the dilute limit, that allow comparison [13,17,23]. From Fig. 3, we see that the experimental dependence of $D$ on $\rho_{0}$ continues to be consistent with 2D DLCA simulations throughout the range of overlap. The observed increase in $D$ with $\rho_{0}$ results from the rapid growth of branches that capture smaller clusters. Since the smaller clusters diffuse in the confines of the branches, they have a greater chance of merging in the interior of the larger structure. For a given radius, structures formed at higher coverage occupy more surface area and consequently have a larger fractal dimension. Comparing geometric scaling properties alone, nanostructures generated by desorption-assisted coalescence are consistent with 2-D DLCA.

\subsection{Aggregation kinetics}

To further compare desorption-assisted coalescence with 2-D DLCA, we can apply DLCA dynamic scaling to BLAG nanostructures. The clustering kinetics of DLCA are described by the time dependence of the weighted average cluster size, $S$, and the total number of clusters, $N$. The weighted average cluster size at time $t$ is defined as $S(t)=\sum s^{2} n_{s}(t) / \sum s n_{s}(t)$, where $n_{s}$ is the number of clusters with a size $s$. Based on DLCA Monte Carlo simulations $[15,16]$, a dynamic scaling function has been proposed that gives a power law increase in time for the weighted average cluster size, $S(t) \sim t^{z}$, and a power law decay in time for the total number of clusters, $N(t) \sim t^{-z}$.

Nanostructures produced by buffer layer desorption are not explicitly time-dependent, but their distribution does depend on $t_{\mathrm{Xe}}$ (the average cluster size increases and the cluster density decreases with increasing $t_{\mathrm{Xe}}$, Fig. 1). Accordingly, we assume that $t_{\mathrm{Xe}}$ is equivalent to time and compare the observed dynamic scaling with DLCA predictions. If there is equivalency, then logarithmic plots of weighted average cluster size and the number density of clusters versus $t_{\mathrm{Xe}}$ should be linear. Fig. 4(a) shows linear behavior for $\ln S\left(t_{\mathrm{Xe}}\right)$ as a function of $\ln t_{\mathrm{Xe}}$ for $20 \leqslant t_{\mathrm{Xe}} \leqslant 215 \mathrm{ML}$ for both 1 and $5 \AA$ Au depositions where $\rho_{0}$ was $\sim 0.04$ and 0.21 , respectively. Fig. 4(b) shows equivalent dependence of $\ln N\left(t_{\mathrm{Xe}}\right)$ on $\ln t_{\mathrm{Xe}}$. Fits to the high and low coverage data yield $z \sim 2$ as the exponent that characterizes the nanostructure dependence on the buffer layer thickness for $\rho_{0} \sim 0.04-0.21$. The dynamic behavior of desorption-assisted coalescence serves as an additional confirmation of its consistency with DLCA.

\section{Summary}

We have used TEM to examine desorption-assisted coalescence of $\mathrm{Au}$ nanostructures on Xebuffered amorphous carbon substrates. To gain insight into the diffusion and capture of the nano- 

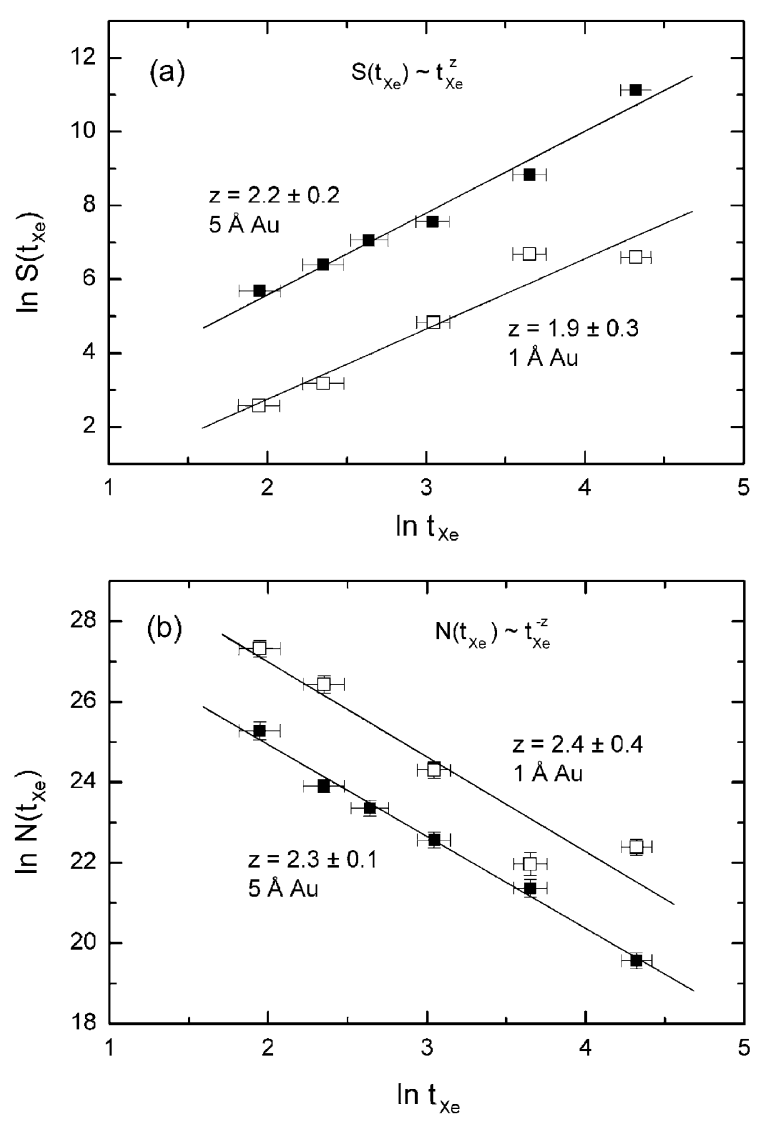

Fig. 4. Kinetics of clustering. The growth of the weighted average cluster size, $S$ (a), and the decay of the average cluster density, $N\left(\right.$ b), as a function of buffer layer thickness, $t_{\mathrm{Xe}}$, for $\mathrm{Au}$ depositions of $1 \AA\left(\rho_{0} \sim 0.04\right)$ and $5 \AA\left(\rho_{0} \sim 0.21\right)$. The linear fits show power law dependences. The exponent that characterizes the nanostructure dependence on the buffer layer is $z \sim 2$.

structures, we determined the fractal dimension for initial surface coverages from $\rho_{0}=0.04(D \sim 1.42)$ to $\rho_{0}=0.21(D \sim 1.72)$. These results are consistent with simulations for 2-D DLCA. Moreover, the results show a strong dependence on the buffer layer thickness, and if we replace time-dependent relationships proposed for DLCA with buffer layer thickness relationships, we find that both the weighted average cluster size and the cluster density show a power law dependence on $t_{\mathrm{Xe}}$ with $z \sim 2$. This exponent should describe the dynamic behavior for coverages below the range studied, but there will be an upper limit to the behavior as the initial coverage approaches the 2-D percolation threshold of $\rho_{0}=0.59$.

The insights gained here will be important in future studies seeking controlled nanostructure growth with BLAG. In particular, with a scaled cluster size distribution, a priori selection of the average cluster size and density will be feasible. Moreover, the dynamic scaling exponent $z$ will be useful in comparing Xe with other buffer layers. Quantitative comparisons may discern whether such systems allow for alternate modes of energy transition through the buffer that could change the initial distribution of clusters and influence coalescence during desorption. It is also interesting to extend these studies to specific systems such as $\mathrm{Pd}$ clusters on a $\mathrm{CO}$ buffer where $\mathrm{CO}$ will chemisorb to the Pd clusters and limit coalescence. In such cases, RLCA may be a better model to describe the geometric and dynamic behavior of cluster coalescence.

\section{Acknowledgements}

This work was supported by the US Department of Energy under contract number DEFG0291ER45439, by the Army Research Office, and by a National Defence Science and Engineering Graduate Fellowship. TEM imaging was carried out in the Center for Microanalysis of Materials of the Frederick Seitz Materials Research Laboratory. We thank V. Antonov for assistance in the $\mathrm{Au} / \mathrm{Xe} /$ graphite STM study.

\section{References}

[1] F. Family, D.P. Landau, Kinetics of Aggregation and Gelation, North-Holland, Amsterdam, 1984.

[2] T.A. Witten, L.M. Sander, Phys. Rev. Lett. 47 (1981) 1400.

[3] P. Meakin, Phys. Rev. Lett. 51 (1983) 1119.

[4] M. Kolb, R. Botet, R. Jullien, Phys. Rev. Lett. 51 (1983) 1123.

[5] P. Meakin, J. Chem. Phys. 83 (1985) 3645.

[6] P. Meakin, R. Jullien, J. Phys. (Paris) 46 (1985) 1543.

[7] F. Martinez-Lopez, M.A. Cabrerizo-Vilchez, R. HildagoAlvarez, Physica A 291 (2001) 1. 
[8] D.A. Weitz, J.S. Huang, M.Y. Lin, J. Sung, Phys. Rev. Lett. 53 (1984) 1657.

[9] S.K. Friedlander, Smoke, Dust, and Haze: Fundamentals of Aerosol Behavior, Oxford University Press, New York, 2000.

[10] D.A. Weitz, M. Oliveria, Phys. Rev. Lett. 52 (1984) 1433.

[11] T. Nakayama, A. Nakahara, M. Matsushita, J. Phys. Soc. Jpn. 64 (1995) 1114.

[12] J.H. Weaver, G.D. Waddill, Science 251 (1991) 1444.

[13] R. Jullien, R. Botet, P.M. Mors, Faraday Discuss Chem. Soc. 83 (1987) 125.

[14] R.C. Ball, D.A. Weitz, T.A. Witten, F. Leyvraz, Phys. Rev. Lett. 58 (1987) 274.

[15] T. Vicsek, F. Family, Phys. Rev. Lett. 52 (1984) 1669.

[16] P. Meakin, T. Vicsek, F. Family, Phys. Rev. B 31 (1985) 564.
[17] M. Kolb, H.J. Herrmann, J. Phys. A 18 (1985) L435.

[18] G.D. Waddill, I.M. Vitomirov, C.M. Aldao, S.G. Anderson, C. Capasso, J.H. Weaver, Z. Liliental-Weber, Phys. Rev. B 41 (1990) 5293.

[19] T.R. Ohno, J.C. Patrin, U.S. Ayyala, J.H. Weaver, Phys. Rev. B 44 (1991) 1891.

[20] L. Huang, S.J. Chey, J.H. Weaver, Phys. Rev. Lett. 80 (1998) 4095.

[21] S.J. Chey, L. Huang, J.H. Weaver, Surf. Sci. 419 (1998) L100.

[22] Y.W. Mo, J. Kleiner, M.B. Webb, M.G. Lagally, Phys. Rev. Lett. 66 (1991) 1998.

[23] St.C. Pencea, M. Dumitrascu, Fractal aspects of materials, in: F. Family, P. Meakin, B. Sapoval, R. Wool (Eds.), Vol. 367 of Materials Research Society Symposium Proceedings, MRS, Pittsburgh, 1995, pp. 373-378. 